\title{
Composite dark matter from a model with composite Higgs boson
}

\author{
Maxim Yu. Khlopov* and Chris Kouvaridt \\ *Center for Cosmoparticle physics "Cosmion", 125047, Moscow, Russia \\ Moscow Engineering Physics Institute, 115409 Moscow, Russia, and \\ APC laboratory 10, rue Alice Domon et Léonie Duquet 75205 Paris Cedex 13, France, \\ †The Niels Bohr Institute, Blegdamsvej 17, DK-2100 Copenhagen, Denmark
}

\begin{abstract}
In a previous paper [1], we showed how the minimal walking technicolor model (WTC) can provide a composite dark matter candidate, by forming bound states between a -2 electrically charged techniparticle and a ${ }^{4} \mathrm{He}^{++}$. We studied the properties of these techni-O-helium $\mathrm{tOHe}$ "atoms", which behave as warmer dark matter rather than cold. In this paper we extend our work on several different aspects. We study the possibility of a mixed scenario where both $t O H e$ and bound states between +2 and -2 electrically charged techniparticles coexist in the dark matter density. We argue that these newly proposed bound states solely made of techniparticles, although they behave as Weakly Interacting Massive Particles (WIMPs), due to their large elastic cross section with nuclei, can only account for a small percentage of the dark matter density. Therefore we conclude that within the minimal WTC, composite dark matter should be mostly composed of tOHe. Moreover in this paper, we put cosmological bounds in the masses of the techniparticles, if they compose the dark matter density. Finally we propose within this setup, a possible explanation of the discrepancy between the DAMA/NaI and DAMA/LIBRA findings and the negative results of CDMS and other direct dark matter searches that imply nuclear recoil measurement, which should accompany ionization.
\end{abstract}

\footnotetext{
*Electronic address: khlopov@apc.univ-paris7.fr

$\dagger$ Electronic address: kouvaris@nbi.dk
} 


\section{INTRODUCTION}

Walking technicolor theories (WTC) have regained a lot of interest recently. This is because they can naturally break the electroweak symmetry without violating experimental constraints by the electroweak precision measurements. Several technicolor theories that have techniquarks transforming under higher representations of the gauge group, require a small number of colors and flavors in order to become quasi-conformal [2, 3, 4, 5]. Because of this property, the Higgs particle can be composed of two techniquarks and be able to couple even to the heavier Standard Model particles like the top quark. On the other hand, the fact that these theories become conformal only for a small number of colors and flavors, differentiates them from the old baroque technicolor models that are excluded by the electroweak precision measurements. In addition, the possibility of unification of the couplings makes the walking technicolor theories legitimate candidates for the Large Hadronic Collider (LHC) [6].

Among the walking technicolor theories, special interest has been drawn to the minimal case. This particular model contains only two techniquarks that transform under the adjoint representation of the $S U(2)$ technicolor group and a new lepton family in order to cancel the Witten global anomaly. This minimal model has been investigated thoroughly in [7, 8]. A holographic description of the theory was presented in [9], where several predictions regarding the mass spectrum were made. Lattice methods have also been used recently for the study of gauge theories with fermions that transform under higher dimensional representations [10, 11, 12]. Although simple in nature, this minimal walking technicolor model can provide several possibilities for dark matter. In particular, the theory can admit as dark matter particles technibaryons [13], bound states between a neutral techniquark and a technigluon [14], heavy leptons of the fourth lepton family [15], or bound states between a -2 electrically charged techniparticle and a $H e^{++}[1]$. In the latter case, WTC offers a new exciting realization of a composite dark matter scenario, which was earlier considered in different aspects in the model of teraparticles [16, 17], in the AC model [18, 19], based on the approach of an almost commutative geometry [20, 21], and in the model of 4th generation [22, 23, 24, 25], assuming existence of stable heavy $U$ quark [26].

In all these recent models (see review in [27, 28, 29, 30]), the predicted stable charged particles form neutral atom-like states, composing the dark matter of the modern Universe 
and escaping experimental discovery. It offers new solutions for the physical nature of the cosmological dark matter. The main problem for these solutions is to suppress the abundance of positively charged species bound with ordinary electrons, which behave as anomalous isotopes of hydrogen or helium. This problem remains unresolved, if the model predicts stable particles with charge -1 , as it is the case for tera-electrons [16, 17].

The possibility of stable doubly charged particles $A^{--}$and $C^{++}$, revealed in the $\mathrm{AC}$ model, offered a candidate for dark matter in the form of elusive (AC)-atoms. In the charge symmetric case, when primordial concentrations of $A^{--}$and $C^{++}$are equal, their binding in the expanding Universe is not complete due to freezing out and a significant fraction of free relic $C^{++}$, which is not bound in (AC)-atoms, is left in the Universe and represents a potential danger of anomalous helium overproduction. The suppression of this fraction in terrestrial matter involves a new long range interaction between $\mathrm{A}$ and $\mathrm{C}$, making them to recombine in (AC)-atoms inside dense matter bodies [18, 19].

In the asymmetric case, corresponding to excess of -2 charge species, as it was assumed for $(\bar{U} \bar{U} \bar{U})$ in the model of stable $U$-quark of a 4 th generation, their positively charged partners annihilate effectively in the early Universe. The dark matter is in the form of nuclear interacting O-helium - atom-like bound states of -2 charged particles and primordial helium, formed as soon as $\mathrm{He}$ is produced in Big Bang Nucleosynthesis (BBN). Such an asymmetric case was realized in [1] in the framework of WTC, where it was possible to find a relationship between the excess of negatively charged anti-techni-baryons and/or technileptons and the baryon asymmetry of the Universe.

The minimal walking technicolor model we use is the same as in our previous paper [1] (and references therein). It contains two techniquarks that transform under the adjoint representation of an $S U(2)$ gauge group, i.e. up $U$ and down $D$, with electric charges 1 and 0 respectively. There is also a new fourth family of leptons $\nu^{\prime}$ and $\zeta$ with charges -1 and -2 respectively. This hypercharge assignment is not unique, however it is consistent, since it makes the theory gauge anomaly free. It was already noticed in [1] that since two types of stable doubly charged particles (technibaryon $(U U)^{++}$and technilepton $\zeta^{--}$) can exist, the excess of positively charged $(U U)^{++}$together with the excess of negatively charged $\zeta^{--}$ is also possible, giving rise to atom-like $[(U U) \zeta]$ WIMP species.

Here we analyze the ability of WTC to provide this WIMP solution for composite dark matter. It is evident that the predicted abundance and cosmological role of $[(U U) \zeta]$ are 
determined by the relation between the excess of its constituents $(U U)^{++}$and $\zeta^{--}$. Their excess can be different, although the case where the excess of $(U U)^{++}$is larger, leads to the unresolved problem of anomalous helium overproduction.

One can find a similar problem in the case where the excess of $(U U)^{++}$is equal to the excess of $\zeta^{--}$. In full analogy with the cosmology of the AC model [18, 19], most of $(U U)^{++}$ and $\zeta^{--}$are bound in this case in $[(U U) \zeta]$ "atoms", but the remaining fraction of unbound $(U U)^{++}$is still up to ten orders of magnitude larger than the experimental upper limits on anomalous helium in terrestrial matter [31]. Since the minimal WTC can not offer new long range interactions between $(U U)$ and $\zeta$, ordinary atoms of anomalous helium $[(U U) e e]$ and nuclear interacting techni-O-helium $\left[\mathrm{He}^{++} \zeta^{--}\right]$, having different mobilities in matter, inevitably fractionate. It prevents their recombination in $[(U U) \zeta]$, which might reduce the concentration of anomalous helium in terrestrial matter below experimental upper limits.

Therefore to solve the problem of anomalous helium in the framework of minimal WTC, we are left with the only option to have the excess of negatively charged $\zeta^{--}$larger than the excess of $(U U)^{++}$. This provides complete binding of $(U U)^{++}$in $\left[(U U)^{++} \zeta^{--}\right]$, while the residual excessive $\zeta^{--}$bind with helium in techni-O-helium. This solution can be effective even if the excess of $\zeta^{--}$exceeds the excess of $(U U)^{++}$by relative amount of $\sim 10^{-8}$. Therefore it seems that the WIMPs $[(U U) \zeta]$ can be the dominant dark matter component, making the nuclear interacting techni-O-helium dynamically negligible, as it was the case for the AC model [18, 19].

However, we'll show here that unlike the neutral $(A C)$ atoms, having zero electroweak charge, the weak charge of $[(U U) \zeta]$ is non-zero and its interaction with nuclei, mediated by ordinary $Z$-boson, should lead to an observable effect in the CDMS experiment [32, 33], unless the contribution of $[(U U) \zeta]$ to the total dark matter density is restricted to be a few percent.

An interesting feature of the considered scenario is that in a wide interval of masses of $(U U)$ and $\zeta$, the generation of excess corresponding to the saturation of the observed dark matter by techniparticles, predicts a fixed negative value for the ratio of lepton number $L$ over the baryon number $B$. This ratio is constant for masses below few $\mathrm{TeV}$ and then rapidly grows by absolute value for larger masses and exceeds $10^{8}$, when they approach $10 \mathrm{TeV}$. A large negative value of $L / B$ corresponds to strong lepton asymmetry and to the excess of antineutrino in the period of $\mathrm{BBN}$, which leads to a corresponding growth of primordial $\mathrm{He}$ 
abundance. This argument provides an upper limit on masses of techniparticles.

The paper is organized as follows. After a brief description of the general chronological framework for the considered techniparticle Universe (Section II), we study the relation between baryon asymmetry and techniparticle excess, fixing the value of $L / B$ ratio (Section III). In Section IV] we deduce an upper limit on possible contributions of $[(U U) \zeta]$ WIMPs in the total dark matter density, which follow from the most recent severe constraints of the CDMS experiment [33]. We also speculate on the possibility to explain the positive results of DAMA/NaI (see for review [34]) and DAMA/Libra [35] experiments by ionization effects of inelastic processes, induced by techni-O-helium in the matter. We consider the main results of the present work in Section $\nabla$.

\section{CHRONOLOGICAL FRAMEWORK OF THE TECHNIPARTICLE UNI- VERSE}

It is well known that strong technicolor interactions provide strong exponential suppression of frozen antitechnibaryons, if a technibaryon excess is generated. Since the technilepton interaction is much weaker, even in the presence of a technilepton excess, the freeze out concentration of positively charged anti-techni-leptons $\bar{\zeta}^{++}$can be significant. However, our previous detailed analysis [1] has shown that in the period after BBN, all the remaining $\bar{\zeta}^{++}$ can be effectively eliminated by techni-O-helium catalysis. This catalysis, taking place after all the free $\zeta^{--}$bind with helium, formed in BBN, provides also an effective binding of all the remaining free $(U U)$ in $[(U U) \zeta]$ WIMPs. The constraint on the contribution of $[(U U) \zeta]$ WIMPs to the total dark matter density, which we deduce in Section IV from the results of CDMS search for WIMPs, makes the dynamical evolution of the considered techniparticle Universe virtually coinciding with the picture of techni-O-helium Universe, studied in [1].

On the above reasons we can avoid a detailed analysis of all the stages of cosmological evolution of techniparticles and give only a brief sketch of this evolution, which serves as a framework for our further discussion of several specific problems.

The thermal history of techniparticles starts with the generation of baryon (and/or lepton) asymmetry in the very early Universe. The mechanism of such generation is not specified in the minimal WTC, but owing to sphaleron processes, this asymmetry is redistributed in the equilibrium, giving rise to the excess of technilepton $L^{\prime}$, technibaryon $T B$, baryon 
and lepton numbers. After the freeze out of the sphaleron processes, these numbers are conserved separately and the excess of technibaryons and technileptons is fixed. It results to the excess of the lightest stable technibaryons $U U^{++}$and technileptons $\zeta^{--}$. Both species behave as charged leptons in particle physics experiments and their absence in accelerator searches puts a lower bound on their mass, about $100 \mathrm{GeV}$. For numerical estimations below we introduce the notation $m_{\zeta}=100 S_{2} \mathrm{GeV}$ for the mass of $\zeta^{--}, m_{U U}=100 B_{2} \mathrm{GeV}$ for the mass of $U U^{++}$and $\mu=m_{U U} m_{\zeta} /\left(m_{U U}+m_{\zeta}\right)=100 R_{2} \mathrm{GeV}$ for the reduced mass of the $U U$ and $\zeta$ system. With the use of this notation, the chronology of techniparticle evolution after the generation of technibaryon and technilepton asymmetry, looks as follows:

1) In the period $10^{-10} S_{2}^{-2} \mathrm{~s} \leq t \leq 6 \cdot 10^{-8} S_{2}^{-2} \mathrm{~s}$ at $m_{\zeta} \geq T \geq T_{f}=m_{\zeta} / 31 \approx 3 S_{2} \mathrm{GeV}, \zeta-$ lepton pairs $\zeta \bar{\zeta}$ annihilate and freeze out. For large $m_{\zeta}$, the abundance of frozen out $\zeta$-lepton pairs is not suppressed in spite of a $\zeta$-lepton excess. A similar period with the exchange of $S_{2}$ by $B_{2}$ can be mentioned for the freeze out of $U U$ and $\bar{U} \bar{U}$ pairs. Due to the strong technicolor interaction, the freeze out abundance of $\bar{U} \bar{U}$ is exponentially small for all the reasonable masses of technibaryons.

Even at the largest possible values of $S_{2}$ and $B_{2}$, the freeze out temperature $T_{f}$ for techniparticles does not exceed substantially the freeze out temperature for sphaleron processes. Because of this, the process of freezing out the technipartcles can not strongly influence the conditions under which techniparticle excess is generated.

2)In the period $6 \cdot 10^{-4} R_{2}^{-2} \mathrm{~s} \leq t \leq 5.4 R_{2}^{-2} 10^{-1} \mathrm{~s}$ at $I_{U \zeta} \approx 40 R_{2} \mathrm{MeV} \geq T \geq I_{U \zeta} / 30$ negatively charged technileptons $\zeta^{--}$can recombine with positively charged technibaryons $U U^{++}$in atom-like atoms $[(U U) \zeta]$ with potential energy $I_{U \zeta}=Z_{U U}^{2} Z_{\zeta}^{2} \alpha^{2} \mu / 2 \approx 40 R_{2} \mathrm{MeV}$ $\left(Z_{U U}=2, Z_{\zeta}=-2\right)$. This process is frozen out at $T \approx I_{U \zeta} / 30$, when the inverse reaction of $[(U U) \zeta]$ photodestruction is not effective to prevent recombination of $U U$ and $\zeta$. Together with neutral $[(U U) \zeta]$ atoms, free charged $\zeta^{--}$and $U U^{++}$are also left, being the dominant form of techniparticle matter at large $R_{2}$.

3)At $t \sim 2.4 \cdot 10^{-3} S_{2}^{-2} \mathrm{~s}$ and the temperature $T \sim I_{\zeta}=20 S_{2} \mathrm{MeV}$, corresponding to the binding energy $I_{\zeta}=Z_{\zeta}^{4} \alpha^{2} m_{\zeta} / 4 \approx 20 S_{2} \mathrm{MeV}\left(Z_{\zeta}=-2\right) \zeta$-positronium "atoms" $\left(\zeta^{--} \bar{\zeta}^{++}\right)$ are formed, in which $\bar{\zeta}^{++}$annihilate. At large $m_{\zeta}$ this annihilation is not at all effective to reduce the $\zeta \bar{\zeta}$ pairs abundance. These pairs are eliminated in the course of the successive evolution of techniparticles.

4)In the period $100 \mathrm{~s} \leq t \leq 300 \mathrm{~s}$ at $100 \mathrm{keV} \geq T \geq T_{o}=I_{o} / 27=8 \alpha^{2} m_{\mathrm{He}} / 27 \approx 60 \mathrm{keV}$, 
${ }^{4} \mathrm{He}$ has already been formed in the $\mathrm{BBN}$ and virtually all free $\zeta^{--}$are trapped by ${ }^{4} H e$ in techni-O-helium "atoms" $\left({ }^{4} \mathrm{He}^{++} \zeta^{--}\right)$. Being formed, techni-O-helium catalyzes the binding of free $U U$ with its constituent $\zeta^{--}$in $[(U U) \zeta]$ atoms and of free $\bar{\zeta}^{++}$into $\zeta$-positronium and completes the annihilation of all the primordial antitechnileptons. At large $m_{\zeta}$, in spite of a significant fraction of free $\bar{\zeta}^{++}$, the effects of $\left(\zeta^{--} \bar{\zeta}^{++}\right)$annihilation catalyzed by techni-O-helium, do not cause any contradiction with observations.

Techni-O-helium, being an $\alpha$-particle with screened electric charge, can catalyze nuclear transformations, which can influence primordial light element abundance and cause primordial heavy element formation. These effects need a special detailed and complicated study. The arguments of [1] indicate that this model does not lead to immediate contradictions with the observational data.

After having been formed, the weakly interacting neutral $U U \zeta$ "atoms" immediately decouple from the plasma, being close to typical cold dark matter particles by spectrum of their density fluctuations.

Due to nuclear interactions of its helium constituent with nuclei in the cosmic plasma, the techni-O-helium gas is in thermal equilibrium with plasma and radiation on the Radiation Dominance (RD) stage, while the energy and momentum transfer from the plasma is effective. The radiation pressure acting on the plasma is then effectively transferred to density fluctuations of the techni-O-helium gas and transforms them in acoustic waves at scales up to the size of the horizon. However, as it was first noticed in [22], this transfer to heavy nuclear-interacting species becomes ineffective before the end of the RD stage and such species decouple from plasma and radiation. Consequently, nothing prevents the development of gravitational instability in the gas of these species. This argument was shown in [1] to be completely applicable to the case of techni-O-helium.

5) At temperature $T<T_{o d} \approx 45 S_{2}^{2 / 3} \mathrm{eV}$, estimated in [1], the energy and momentum transfer from baryons to techni-O-helium is not effective because $n_{B}\langle\sigma v\rangle\left(m_{p} / m_{o}\right) t<1$, where $m_{o}$ is the mass of the $t O H e$ atom, $m_{p}$ is the mass of the proton, and $S_{2}=\frac{m_{o}}{100 \mathrm{GeV}}$. Here

$$
\sigma \approx \sigma_{o} \sim \pi R_{o}^{2} \approx 10^{-25} \mathrm{~cm}^{2}
$$

where $R_{o}$ is the size of techni-O-helium, $n_{B}$ is the baryon number density, and $v=\sqrt{2 T / m_{p}}$ is the baryon thermal velocity. The techni-O-helium gas decouples from the plasma and plays the role of dark matter, which starts to dominate in the Universe at $T_{R M}=1 \mathrm{eV}$. 
6) Therefore in the period after $t \sim 10^{12} \mathrm{~s}$ at $T \leq T_{R M} \approx 1 \mathrm{eV}$, the techniparticle dominance starts. Due to the CDMS constraints [33] (see Section IV]), the allowed fraction of $U U \zeta$ is small and the techni-O-helium "atoms" play the main dynamical role in the development of gravitational instability, triggering the large scale structure formation. The composite nature of techni-O-helium determines the specifics of the corresponding dark matter scenario.

The total mass of the $t O H e$ gas with density $\rho_{d}=\frac{T_{R M}}{T_{o d}} \rho_{t o t}$ within the cosmological horizon $l_{h}=t$ is

$$
M=\frac{4 \pi}{3} \rho_{d} t^{3}
$$

In the period of decoupling $T=T_{\text {od }}$, this mass depends strongly on the techniparticle mass $S_{2}$ and is given in [1]

$$
M_{o d}=\frac{T_{R M}}{T_{o d}} m_{P l}\left(\frac{m_{P l}}{T_{o d}}\right)^{2} \approx 2 \cdot 10^{46} S_{2}^{-8 / 3} \mathrm{~g}=10^{13} S_{2}^{-2} M_{\odot},
$$

where $M_{\odot}$ is the solar mass, and $m_{P l}$ is the Planck mass. The techni-O-helium is formed only at $T_{o}$ and its total mass within the cosmological horizon in the period of its creation is $M_{o}=M_{o d}\left(T_{o d} / T_{o}\right)^{3}=10^{37} \mathrm{~g}$.

On the RD stage before decoupling, the Jeans length $\lambda_{J}$ of the $t O H e$ gas was restricted from below by the propagation of sound waves in plasma with a relativistic equation of state $p=\epsilon / 3$, being of the order of the cosmological horizon and equal to $\lambda_{J}=l_{h} / \sqrt{3}=t / \sqrt{3}$. After decoupling at $T=T_{o d}$, it falls down to $\lambda_{J} \sim v_{o} t$, where $v_{o}=\sqrt{2 T_{o d} / m_{o}}$. Though after decoupling the Jeans mass in the $\mathrm{tOHe}$ gas correspondingly falls down

$$
M_{J} \sim v_{o}^{3} M_{o d} \sim 3 \cdot 10^{-14} M_{o d}
$$

one should expect a strong suppression of fluctuations on scales $M<M_{o}$, as well as adiabatic damping of sound waves in the RD plasma for scales $M_{o}<M<M_{o d}$. It can provide some suppression of small scale structure in the considered model for all reasonable masses of techniparticles. The significance of this suppression and its effect on the structure formation needs a special study in detailed numerical simulations. In any case, it can not be as strong as the free streaming suppression in ordinary Warm Dark Matter scenarios, but one can expect that qualitatively we deal with a Warmer Than Cold Dark Matter model. Being decoupled from baryonic matter, the $\mathrm{tOHe}$ gas does not follow the formation of baryonic 
astrophysical objects (stars, planets, molecular clouds...) and forms dark matter halos of galaxies.

Based on this general framework, we analyze the generation of techniparticle excess and the results of direct dark matter searches, which fix the parameters of the considered scenario and lead to robust predictions.

\section{CALCULATION OF TECHNIPARTICLE EXCESS}

In this section we calculate the relic densities of the particles of interest. Our derivation is similar as in [1, 13]. We assume the existence of a baryon-antibaryon asymmetry created sometime after inflation. Equilibrium between ordinary and techni-particles, supported by WTC, provides a definite relation between this asymmetry and technibaryonantitechnibaryon asymmetry. In addition, we assume that the conditions of thermal equilibrium via weak interactions hold down to the temperature where the sphalerons freeze out. Under these conditions, the ratio of the technibaryon number $T B$ over the baryon number $B$ is given as

$$
\frac{T B}{B}=-\sigma_{U U}\left(\frac{L^{\prime}}{B} \frac{1}{3 \sigma_{\zeta}}+1+\frac{L}{3 B}\right),
$$

where $L$ and $L^{\prime}$ are the lepton number and the technilepton number respectively. The parameters $\sigma_{U U}$ and $\sigma_{\zeta}$ are statistical factors that depend on the mass of the particle in question and the freeze out temperature of the sphalerons and are defined in [1]. We should also mention that the above equation holds under the condition of a second order phase transition for the electroweak symmetry. The results for a first order phase transition has been shown not to change the conclusions significantly [13].

In [1], we examined the possibility that negatively -2 charged particles with substantial abundance can bind with ${ }^{4} \mathrm{He}$ forming electrically neutral atoms that can play the role of strongly interacting massive particles (SIMP) as part of warm dark matter. In particular, we investigated the possibility of $H e \bar{U} \bar{U}$ and $H e \zeta$ atoms. In this paper, we continue our investigation by looking at the possibility that $U U^{++}$can bind with $\zeta^{--}$forming again a neutral atom that can contribute to the dark matter density. As we are going to argue, such a bound state differs qualitatively from the $H e \zeta$ and $H e \bar{U} \bar{U}$ states, since it behaves more as a WIMP, rather than a SIMP. As it has been argued previously [1, 13], both $U U$ and $\zeta$ carry technibaryon $T B$ and technilepton number $L^{\prime}$ respectively. If no interactions violate 
$T B$ and $L^{\prime}$, and if $U U$ and $\zeta$ are the lightest technibaryon and technilepton respectively, these particles are absolutely stable. Here, we investigate the possibility that there is a substantial relic density for $U U$ and $\zeta$, with the density of $\zeta$ being higher than that of $U U$. In this case, the overwhelming majority of $U U$ will be captured by $\zeta$ forming neutral bound states $U U \zeta$, while the remaining $\zeta$ will be captured by ${ }^{4} H e^{++}$, forming neutral $H e \zeta$. In such a case, the contribution to the dark matter density $\Omega_{d}$ is

$$
\frac{\Omega_{d}}{\Omega_{B}}=\frac{\Omega_{T B}}{\Omega_{B}}+\frac{\Omega_{L^{\prime}}}{\Omega_{B}}=\frac{3}{2} \frac{T B}{B} \frac{m_{d}}{m_{p}}+\left(\frac{L^{\prime}}{B}-\frac{3}{2} \frac{T B}{B}\right) \frac{m_{\zeta}}{m_{p}},
$$

where $m_{d}$ is the mass of the bound state of $U U \zeta, m_{p}$ is the mass of the proton, and $m_{\zeta}$ is the mass of the bound state $H e \zeta$. If we denote by $x$ the portion of dark matter composed of $U U \zeta$ (and therefore $1-x$ the one made of $H e \zeta$ ), by using Eq. (3), we get the ratio $L / B$ as a function of $x$ as

$$
\frac{L}{B}=-3-5 m_{p}\left(\frac{2 x}{m_{d} \sigma_{U U}}+\frac{x}{m_{d} \sigma_{\zeta}}+\frac{1-x}{m_{\zeta} \sigma_{\zeta}}\right) .
$$

For our scenario to be realized, the quantity inside the parenthesis of Eq. (3) must be negative, in order to have abundance of $U U$ and not $\bar{U} \bar{U}$, while the quantity inside the parenthesis of Eq. (4) should be positive, in order to have more $\zeta$ that $U U$. These two constraints are satisfied by Eq. (5). As we shall argue in the next section, because of the strict CDMS constraints, $U U \zeta$ cannot be more than 4 to $6 \%$ of the dark matter density. The overwhelming amount of dark matter in this case comes from $\mathrm{He} \zeta$. The result for the ratio $L / B$ is quite interesting. For a large range of the parameters, the second term in the right hand side of Eq. (5) is much smaller than -3 . This means that our scenario makes a prediction for the ratio of leptons over the baryons, independent of the specifics of the walking technicolor model, i.e. the masses of the yet unknown hypothetical particles $\zeta$ and $U U$, as well as other model dependent parameters like the freeze out temperature of the sphalerons.

In the upper left panel of Fig. 1, we show the predicted ratio of $L / B$ as a function of the mass $m_{\zeta}$, if dark matter is made only of bound states of $\mathrm{He} \zeta$. In this case the mass of $U U$ is irrelevant. We can see that the ratio $L / B$ is between -5 to -3 independently of the values of $m_{U U}$ and $m_{\zeta}$ as long as the latter does not exceed roughly 2 to $2.5 \mathrm{TeV}$. The predicted value for $L / B$ deviates rapidly from -3 as soon as the mass of $\zeta$ becomes larger than roughly 2 to $2.5 \mathrm{TeV}$. In the upper right panel of Fig. 1 , we show the $L / B$ ratio (again with $x=0$ ) 

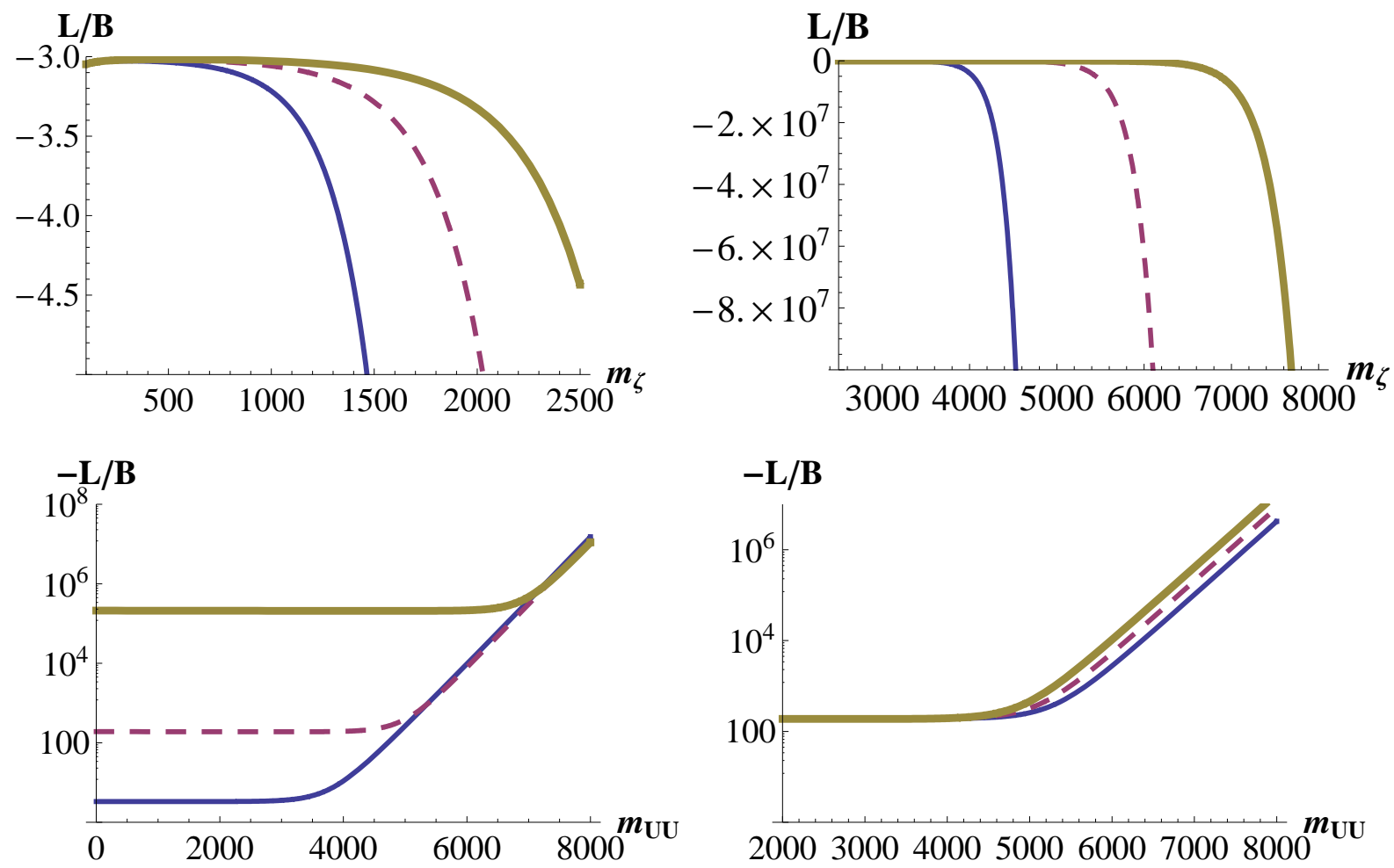

FIG. 1: : The ratio $L / B$ derived from Eq. (5). In the upper panels, $L / B$ is plotted as a function of $m_{\zeta}$, up to $2.5 \mathrm{TeV}$ for the left panel and from 2.5 to $10 \mathrm{TeV}$ for the right one. $x=0$ (there is no $U U \zeta$ dark matter density). The thin solid, dashed and thick solid lines correspond respectively to sphaleron freeze out temperatures of 150,200 , and $250 \mathrm{GeV}$. In the lower left panel, we plot the absolute value of $L / B$ in a logarithmic scale as a function of $m_{U U}$ if $U U \zeta$ makes up $3 \%$ of dark matter and sphaleron freeze out temperature is $250 \mathrm{GeV}$, for $m_{\zeta}=2 \mathrm{TeV}$ (thin solid line), $4 \mathrm{TeV}$ (dashed line), and $6 \mathrm{TeV}$ (thick solid line). In the lower right panel, we plot the same ratio as in the lower left panel having fixed $m_{\zeta}=4 \mathrm{TeV}$, for three different values of $x, 1 \%$ (thin solid line), $2 \%$ (dashed line), and $4 \%$ (thick solid line).

for $m_{\zeta}$ up to $10 \mathrm{TeV} . L / B$ increases (as an absolute value) exponentially for large values of $m_{\zeta}$. This is due to the fact that for large values of $m_{\zeta}$, the statistical factor $\sigma_{\zeta}$ becomes exponentially small. Therefore, the second term in the right hand side of Eq. (5) becomes much larger than -3 . Although one would expect $U U$ and $\zeta$ to have masses of the order of the electroweak scale, due to lack of tools to handle the non-perturbative dynamics of the technicolor model, at the moment nothing forbids the masses to be even several TeV. $L / B$ becomes of the order of $\sim-10^{8}$ for $m_{\zeta}$ equal to roughly $4.5,6$ and $7.5 \mathrm{TeV}$ for sphaleron 
freeze out temperatures 150, 200 and $250 \mathrm{GeV}$ respectively. Cosmological constraints forbid larger negative values for $L / B$ [36] and therefore this constrains $m_{\zeta}$.

Now we turn our interest to nonzero $x$, which means that there is a small portion of WIMP type $U U \zeta$ dark matter. In the lower left panel of Fig. 1, we plot the absolute value of $L / B$ in a logarithmic scale as a function of $m_{U U}$ for the case where $U U \zeta$ makes up $3 \%$ of the total dark matter density, for three different values of $m_{\zeta} 2,4$, and $6 \mathrm{TeV}$. In the lower right panel of Fig. 1, we plot the same ratio for a fixed $m_{\zeta}=4 \mathrm{TeV}$, and three different values of $x$, i.e. $1 \%, 2 \%$, and $4 \%$.

\section{DETECTION OF COMPOSITE STATES WITH TECHNIPARTICLES}

\section{A. Detection of UU $\zeta$}

The neutral bound state between an electrically positively +2 charged $U U$ and a negatively -2 charged $\zeta$ has completely different features as a dark matter candidate from $\mathrm{He} \zeta$ and the techni-O-helium candidates presented in [1]. In the case of $\mathrm{He} \zeta$ (and in general for all techni-O-helium candidates), because of the $\mathrm{He}$ atom, the elastic cross section with nuclei is very large (of the order of $10^{-25} \mathrm{~cm}^{2}$ ). If such a particle exists, the large cross section with nuclei will slow down the particle sufficiently in case it enters the atmosphere of the Earth, that the recoil energy in the underground based detectors like CDMS will be below the required threshold [1]. Only balloon, ground, or space based detectors can possibly detect this particle. On the other hand, $U U \zeta$ does not contain helium nucleus and has an elastic cross section with nuclei much smaller than $H e \zeta$. As we shall argue, the elastic cross section is effectively the same as of a heavy Dirac neutrino. This means that CDMS constraints should be taken into consideration, since $U U \zeta$ behaves as a typical WIMP.

The elastic spin independent cross section of a neutral particle scattering off nuclei targets is

$$
\sigma_{0}=\frac{G_{F}^{2}}{2 \pi} \mu^{2} Y^{2} \bar{N}^{2} F^{2}
$$

where $G_{F}$ is the Fermi constant, $Y$ is the weak hypercharge of the WIMP and $\mu$ is the reduced mass of the WIMP and the target nucleus. $\bar{N}=N-\left(1-4 \sin ^{2} \theta_{w}\right) Z$, where $N$ and $Z$ are the number of neutrons and protons in the target nucleus and $\theta_{w}$ is the Weinberg angle. The parameter $F^{2}$ is a form factor for the target nucleus. The cross section can be 
written as

$$
\sigma_{0}=8.44 \times 10^{-3} \mu^{2} Y^{2} \bar{N}^{2} F^{2} \mathrm{pb}
$$

In this case, our WIMP is not a single neutral particle, but it is a bound state between two charged particles $U U^{++}$and $\zeta^{--}$. The Bohr radius of such a bound state is of the order of $10^{-15} \mathrm{~cm}$ for typical masses $m_{U U}$ and $m_{\zeta}$ of the order of $\mathrm{TeV}$. For recoil energies of the order of $10 \mathrm{keV}$, the $Z$ boson that mediates the energy between the WIMP and the nucleus has a wavelength which is of the same order of magnitude as the Bohr radius of $U U \zeta$. This means that $Z$ interacts effectively with the whole $U U \zeta$ and not with the constituent particles $U U$ and $\zeta$. The "effective" hypercharge of $U U \zeta$ should be the sum of the corresponding hypercharges of $U U$ and $\zeta$. The $U U$ has hypercharge +1 since it belongs to the triplet of $U U, U D$, and $D D$. The hypercharge of $\zeta$ is $-3 / 2$. Therefore the "effective" hypercharge of $U U \zeta$ is $Y=-1 / 2$. The $G e$ detectors give the most strict constraints in CDMS so far. For a Ge detector, $\bar{N}=38.59$.

For the form factor $F^{2}$, we use the Helm form factor

$$
F^{2}(q)=\left(\frac{3 j_{1}\left(q R_{1}\right)}{q R_{1}}\right)^{2} e^{-q^{2} s^{2}}
$$

where $q=\sqrt{2 M_{n} T}$ is the recoil momentum of the target nucleus, $T$ is the recoil energy, $M_{n}$ is the mass of the target nucleus, and $j_{1}\left(q R_{1}\right)$ is the spherical Bessel function. The parameter $s=0.9 \mathrm{fm}$ and $R_{1}$ is defined through

$$
R_{1}=\sqrt{c^{2}+7 \pi^{2} a^{2} / 3-5 s^{2}}
$$

where $a=0.52 \mathrm{fm}$ and $c \simeq 1.23 A^{1 / 3}-0.6 \mathrm{fm}$. For $G e A=73$. The number of projected counts in CDMS is given by

$$
\text { counts }=\int_{E_{1}}^{E_{2}} \frac{d R}{d T} d T \times \tau,
$$

where $\tau$ is the exposure of the detector measured in kg.days, $E_{1}$ and $E_{2}$ are respectively the lower and upper bounds for the recoil energy that the detectors have satisfying efficiency. We take $E_{1}=10 \mathrm{keV}, E_{2}=100 \mathrm{keV}$, and $\tau=121 \mathrm{~kg}$.days. The differential rate with respect to the recoil energy is

$$
\frac{d R}{d T}=c_{1} \frac{R_{0}}{E_{0} r} e^{-c_{2} T / E_{0} r}
$$




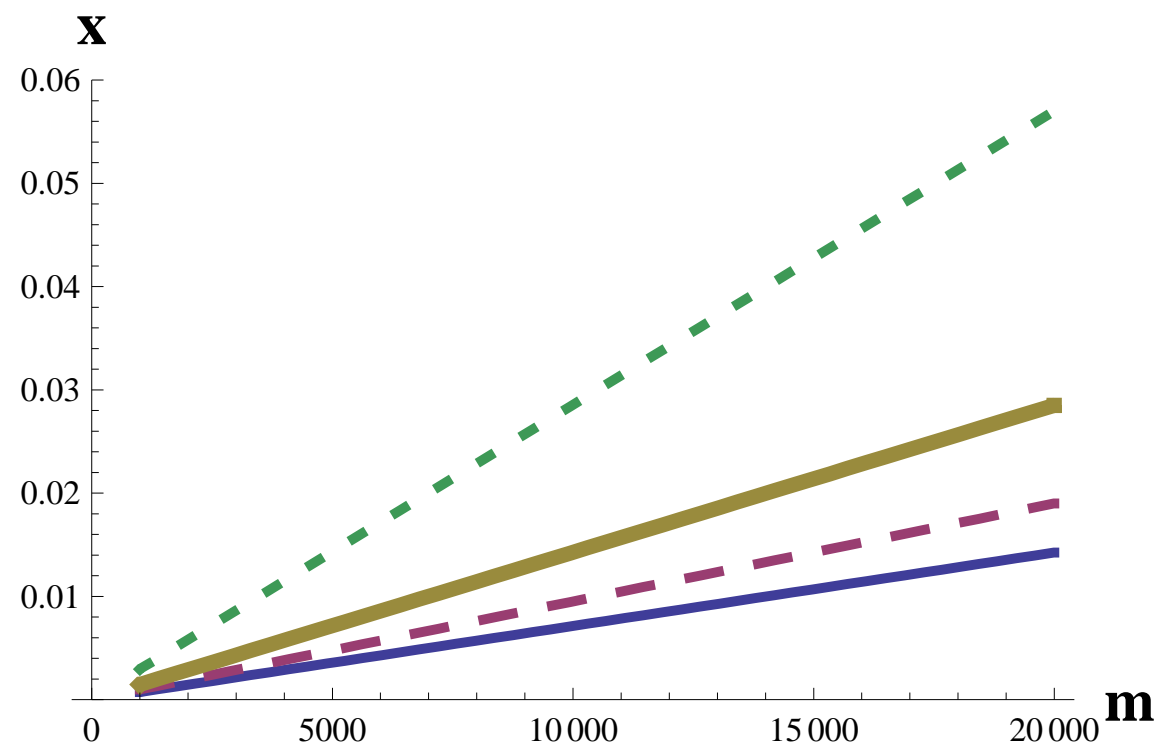

FIG. 2: The upper limit of the contribution of $U U \zeta$ to the dark matter density as a function of its mass. The four lines correspond to different local dark matter densities for the Earth, namely $0.1 \mathrm{GeV} / \mathrm{cm}^{3}$ (dotted line), $0.2 \mathrm{GeV} / \mathrm{cm}^{3}$ (thick solid line), $0.3 \mathrm{GeV} / \mathrm{cm}^{3}$ (dashed line), and 0.4 $\mathrm{GeV} / \mathrm{cm}^{3}$ (thin solid line).

where $E_{0}$ is the kinetic energy of the WIMP, and $r=4 m M_{n} /\left(m+M_{n}\right)^{2}, m$ being the mass of the WIMP. The constants $c_{1}=0.751$, and $c_{2}=0.561$, are fitting parameters that take into account the motion of the Earth [37]. The parameter $R_{0}$ is

$$
R_{0}=\frac{503}{M_{n} m}\left(\frac{\sigma_{0}}{1 \mathrm{pb}}\right)\left(\frac{\rho_{d m}}{0.4 \mathrm{GeVc}^{-2} \mathrm{~cm}^{-3}}\right)\left(\frac{v_{0}}{230 \mathrm{kms}^{-1}}\right) \mathrm{kg}^{-1} \mathrm{days}^{-1}
$$

The parameters $\rho_{d m}$ and $v_{0}$ are the local dark matter density of the Earth and the thermal velocity of the WIMP respectively. It is understood that since in our scenario $U U \zeta$ makes up $x 100 \%$ of the total dark matter density, $\rho_{d m}$ should always be multiplied by $x$. Here, we are going to use $v_{0}=220 \mathrm{~km} / \mathrm{s}$. We should also mention that the $90 \%$ confidence level for a confirmed event is 2.3 counts. So far, no confirmed counts have been found in CDMS [32, 33]. As Fig. 2 shows, the CDMS constraints restrict severely the percentage of $U U \zeta$ in the dark matter. The area above the curves has been excluded by CDMS. For a hypothetical $U U \zeta$ with a mass of $20 \mathrm{TeV}$, this particle can only make up for 3 to $6 \%$ of the dark matter density (if the local dark matter density of the Earth is between 0.1 and $0.3 \mathrm{GeV} / \mathrm{cm}^{3}$ ). A higher portion of $U U \zeta$ is allowed by the theory, but because of the spin independent cross 
section with nuclei, it would have given a clean signal in CDMS. This percentage of course depends strongly on the local dark matter density and the mass of $U U \zeta$. The mass of $U U \zeta$ is roughly speaking the sum of $m_{U U}$ and $m_{\zeta}$ and therefore for a $U U \zeta$ mass of $20 \mathrm{TeV}$, it might correspond to particles $U U$ and $\zeta$ with masses of $10 \mathrm{TeV}$. Under the circumstances, $U U \zeta$ can be only a tiny fraction of dark matter and this means that the rest of dark matter should be $\mathrm{He \zeta}$. For reasons that have been presented in [1], He $\mathrm{H}$ is not detectable in the detectors of CDMS. As we already mentioned masses of $U U$ and/or $\zeta$ larger than 4.5 to 7.5 $\mathrm{TeV}$ (depending on the sphaleron freeze out temperature) give an absolute value for the ratio $L / B$ larger than $10^{8}$ and this might induce problems [36]. The reason the curves in Fig. 2 are almost straight lines has to do with the fact that for large masses of $U U \zeta, R_{0} \sim 1 / m$, $E_{0} \sim m$, and $r \sim 1 / m$. From Eqs. (10), (11), and (12), we see that the mass of $U U \zeta$ is proportional to the dark matter density for a fixed number of counts.

Apart from the CDMS constraint regarding the mass of $U U$ and $\zeta$, there is also a cosmological one. The existence of bound states of $\mathrm{He \zeta}$ reduces the abundance of free $\mathrm{He}^{4}$ in the Universe. The Standard Big Bang Nucleosynthesis (SBBN) predicts roughly 25\% abundance of $\mathrm{He}$ among the baryons. The presence of $H e \zeta$ states requires that a portion of the free helium will be captured by $\zeta$ in order to form $H e \zeta$. If the particle $\zeta$ is sufficiently light and if $\mathrm{He} \zeta$ represents a significant fraction of dark matter, a big amount of $\mathrm{He}$ will be captured, making the overall abundance of free helium much smaller than the predicted (and observationally verified) 25\%. The existence of systematic errors regarding the helium abundance observed in the Universe is a legitimate possibility [38, 39]. For the derivation of our constraint, we are going to assume that the helium abundance can be at most $\pm 2 \%$ away from the SBBN predicted value. Upon making this assumption, we can derive a lower bound for $m_{\zeta}$ as a function of the percentage of $\mathrm{He} \zeta$ in the overall dark matter density. It is understood that the lighter $\zeta$ is, (given a fixed mass density of $\mathrm{He} \zeta$ ), the larger the number density of the bound states of $\mathrm{He} \zeta$ becomes and therefore the amount of $\mathrm{He}$ needed to form $H e \zeta$. This means that the abundance of free $H e$ drops. The constraint comes exactly from the fact that we do not allow a $\mathrm{He}$ abundance decrease more that $2 \%$. The densities of $\mathrm{He} \zeta$ $n_{h e \zeta}$ and $H e n_{H e}$ are respectively

$$
\begin{gathered}
\frac{\Omega_{H e \zeta}}{\Omega_{B}}=5.5 y \Leftrightarrow n_{H e \zeta} m_{H e \zeta}=5.5 y \Omega_{B}, \\
\frac{\Omega_{H e}}{\Omega_{B}}=0.25 \Leftrightarrow n_{H e} m_{H e}=0.25 \Omega_{B},
\end{gathered}
$$


where $y=1-x$ represents the fraction of $\mathrm{He \zeta}$ in the overall dark matter density. The amount of $H e$ captured by $\zeta$ is equal to $n_{H e \zeta}$ and according to the constraint it should not be more than $2 \%$ of the overall baryon mass density. Therefore, if we take the ratio of Eqs. (13) and (14), we have

$$
\frac{n_{H e \zeta}}{n_{H e}}=\frac{5.5 y}{0.25} \frac{m_{H e}}{m_{H e \zeta}}=\frac{2 \%}{25 \%} .
$$

From this equation we can find the lowest $m_{\zeta}$ as a function of $y$ that satisfies the constraint. The mass of $\mathrm{He} \zeta$ is roughly equal to the sum of $m_{H e}$ and $m_{\zeta}$, since the binding energy of $H e \zeta$ is orders of magnitude smaller than either one of them. From the CDMS constraint we argued that $U U \zeta$ cannot account for more than 4 to $6 \%$ of dark matter. This means that $\mathrm{He} \zeta$ has to account for the rest of the dark matter density. For an $100 \%$ component of $\mathrm{He} \zeta$, the lowest $m_{\zeta}=1022 \mathrm{GeV}$. If $\mathrm{He}$ is a component of dark matter, this limit goes down accordingly. In any case, a $\zeta$ heavier than roughly $1 \mathrm{TeV}$, no matter what is the amount of $\mathrm{He} \zeta$ in the dark matter density, change only slightly the abundance of free $\mathrm{He}$ and is consistent with the SBBN.

\section{B. Detection of techni-O-helium}

The constraint on WIMP-like $U U \zeta$ component of dark matter leads to the scenario of techni-O-helium Universe, described earlier in [1]. The composite nature of this dominant fraction of techniparticle dark matter can lead to a number of observable effects.

The nuclear interaction of techni-O-helium with cosmic rays gives rise to ionization of this bound state in the interstellar gas and to acceleration of free $\zeta^{--}$in the Galaxy. Assuming a universal mechanism of cosmic ray acceleration, the anomalous low $Z / A$ component of -2 charged technileptons can be present in cosmic rays and be within the reach for PAMELA and AMS02 cosmic ray experiments.

It should be noted that techni-O-helium is not initially present in significant amounts inside stars so that the injection of free $\zeta$ from Supernova explosions might be suppressed, making the regular mechanisms of cosmic ray acceleration ineffective for this component. Then the $\zeta^{--}$component may have such a low momentum that it can be completely suppressed by Solar modulations and can not penetrate heliosphere. On the other hand, at the stage of red supergiant with size $\sim 10^{15} \mathrm{~cm}$ during the period $\sim 3 \cdot 10^{15}$ s of this stage, up to 
$\sim 10^{-9}$ of atoms of techni-O-helium per nucleon can be captured and give the corresponding fraction in cosmic rays, accelerated by regular mechanisms.

Inelastic interaction of techni-O-helium with the matter in the interstellar space can give rise to radiation in the range from few $\mathrm{keV}$ to few $\mathrm{MeV}$. Though our first estimate shows that such a radiation is below the cosmic nonthermal electromagnetic background radiation observed in this range, special analysis of this effect is of interest.

The evident consequence of the techni-O-helium dark matter is its inevitable presence in the terrestrial matter. This is because terrestrial matter appears opaque to $\mathrm{tOHe}$ and stores all its in-falling flux.

If the $\mathrm{tOHe}$ diffusion in matter is determined by elastic collisions, the in-falling $\mathrm{tOHe}$ particles are effectively slowed down after they fall down terrestrial surface. Then they drift, sinking down towards the center of the Earth, with velocity

$$
V=\frac{g}{n \sigma v} \approx 8 S_{2} A^{1 / 2} \mathrm{~cm} / \mathrm{s}
$$

where $A \sim 30$ is the average atomic weight in terrestrial surface matter, $n=2.4 \cdot 10^{24} / A$ is the number of terrestrial atomic nuclei, $\sigma v$ is the rate of nuclear collisions and $g=980 \mathrm{~cm} / \mathrm{s}^{2}$.

Near the Earth's surface, the techni-O-helium abundance is determined by the equilibrium between the in-falling and down-drifting fluxes. Such neutral $\left({ }^{4} \mathrm{He}^{++} \zeta^{--}\right)$"atoms" may provide a catalysis of cold nuclear reactions in ordinary matter (much more effectively than muon catalysis). This effect needs a special and thorough investigation. On the other hand, $\zeta^{--}$capture by nuclei heavier than helium [40], can lead to production of anomalous isotopes, but the arguments presented in [1], indicate that their abundance should be below the experimental upper limits.

It should be noted that the nuclear cross section of the techni-O-helium interaction with matter escapes the severe constraints [41] on strongly interacting dark matter particles (SIMPs) [41, 42] imposed by the XQC experiment [43]. Therefore, a special strategy of techni-O-helium search is needed, as it was proposed in [44].

In underground detectors, $\mathrm{tOHe}$ "atoms" are slowed down to thermal energies and give rise to energy transfer $\sim 2.5 \cdot 10^{-3} \mathrm{eVA} / S_{2}$, far below the threshold for direct dark matter detection. It makes this form of dark matter insensitive to the CDMS constraints. However, tOHe induced nuclear transformation can result in observable effects.

At a depth $L$ below the Earth's surface, the drift timescale is $t_{d r} \sim L / V$, where $V \sim$ 
$40 S_{2} \mathrm{~cm} / \mathrm{s}$ is given by Eq. (16). It means that the change of the incoming flux, caused by the motion of the Earth along its orbit, should lead at the depth $L \sim 10^{5} \mathrm{~cm}$ to the corresponding change in the equilibrium underground concentration of $\mathrm{OH}$ on the timescale $t_{d r} \approx 2.5$. $10^{3} S_{2}^{-1}$ s. Such rapid adjustment of local fraction of $t O H e$ provides annual modulations of inelastic processes inside the bodies of underground dark matter detectors.

One can expect two kinds of inelastic processes in the matter, composed of atoms with nuclei $(A, Z)$, having atomic number $A$ and charge $Z$

$$
(A, Z)+(H e \zeta) \rightarrow(A+4, Z+2)+\zeta^{--}
$$

and

$$
(A, Z)+(H e \zeta) \rightarrow\left[(A, Z) \zeta^{--}\right]+H e .
$$

The first reaction is possible, if the masses of the initial and final nuclei satisfy the energy condition

$$
M(A, Z)+M(4,2)-I_{o}>M(A+4, Z+2),
$$

where $I_{o}=1.6 \mathrm{MeV}$ is the binding energy of techni-O-helium and $M(4,2)$ is the mass of the ${ }^{4} \mathrm{He}$ nucleus. It is more effective for lighter nuclei, while for heavier nuclei the condition (19) is not valid and reaction (18) should take place.

Both types of energy release processes are of the order of $\mathrm{MeV}$, which seems to have nothing to do with the signals in the DAMA experiment. However, in the reaction (18) such energy is rapidly carried away by the He nucleus, while in the remaining compound state of $\left[(A, Z) \zeta^{--}\right]$, the charge of the initial $(A, Z)$ nucleus is reduced by 2 units and the corresponding transformation of electronic orbits with possible emission of two excessive electrons should take place. The energy difference between the lowest lying $1 s$ level of the initial nucleus with the charge $Z$ and the respective levels of its compound system with $\zeta^{--}$ is given by

$$
\Delta E=Z^{2} \alpha^{2} m_{e} / 2-(Z-2)^{2} \alpha^{2} m_{e} / 2 \approx Z \alpha^{2} m_{e}
$$

It is interesting that the energy release in such a transition for two $1 s$ electrons in ${ }^{53} I_{127}$ is about $2 \mathrm{keV}$, while for ${ }^{81} \mathrm{Tl}_{205}$ it is about $4 \mathrm{keV}$. Taking into account that the signal in the DAMA experiment was detected with similar energy of ionization, this idea deserves more detailed analysis, which might be useful for interpretation of this experiment. Since the experimental cuts in the CDMS experiment, exclude events of pure ionization, which 
are not accompanied by phonon signal, if valid, the proposed mechanism could explain the difference in the results of DAMA and CDMS.

\section{DISCUSSION}

In this paper we explored the cosmological implications of a walking technicolor model with doubly charged technibaryons $U U^{++}$and technileptons $\zeta^{--}$. We studied a possibility for a WIMP-like composite dark matter in the form of heavy "atoms" $\left[U U^{++} \zeta^{--}\right]$. To avoid overproduction of anomalous isotopes (related to $U U^{++}$, which are not bound in these atoms), the excess of -2 charged technileptons $\zeta^{--}$should be larger than the excess of $U U^{++}$generated in the Universe. The residual doubly charged $\zeta^{--}$bind with ${ }^{4} H e$ in the techni-O-helium neutral states.

In all the previous realizations of composite dark matter scenarios, this excess was put by hand to saturate the observed dark matter density. In our paradigm, the abundance of techibaryons and technileptons is connected naturally to the baryon relic density. Moreover, in a rather wide window of techniparticle masses below few $\mathrm{TeV}$, a robust prediction follows for the ratio $L / B$ of lepton and baryon asymmetries. At further increase of techniparticle mass, this ratio grows rapidly. It provides an upper limit on the mass of techniparticles from the condition that large negative value of $L / B$ does not lead to overproduction of primordial ${ }^{4} \mathrm{He}$ in $\mathrm{BBN}$.

Since techni-O-helium binds some fraction of ${ }^{4} \mathrm{He}$, an interesting possibility appears that is at large values of $L / B$, the excessive ${ }^{4} H e$ is hidden in the techni-O-helium. However, due to the non-zero weak isospin charge of $\left[U U^{++} \zeta^{--}\right]$, the presence of this dark matter component should lead to observable effect in underground dark matter detectors. The CDMS constraints reduce the allowed fraction of this component to a few per cent, making techni-O-helium the dominant form of composite dark matter in the considered scenario. On that reason, a possibility to hide the excessive ${ }^{4} \mathrm{He}$ in the techni-O-helium is elusive. On the contrary, even having taken into account possible systematic errors in the determination of primordial helium, to provide its abundance within the observed limits, one should constraint the amount of helium bound with $\zeta^{--}$. Since this amount is determined by the techni-Ohelium number density, the condition that techni-O-helium saturates the observed dark matter density leads to a lower limit for the mass of $\zeta^{--}$. 
We come to the conclusion that in the minimal WTC model, contrary to the case of the AC-model, WIMP-like component of composite atom-like dark matter should be sparse, so that the formation of large scale structure should follow a warmer than cold dark matter scenario of the techni-O-helium Universe considered earlier.

In addition to the detailed description of a warmer than cold dark matter model, another challenging problem that is left for future work is the nuclear transformations catalyzed by techni-O-helium. The question about their consistency with observations remains open since special nuclear physics analysis is needed to reveal what are the actual techni-O-helium effects in BBN and in terrestrial matter.

The latter effects inside the body of underground dark matter detectors can experience annual modulation and lead to ionization events with a few $\mathrm{keV}$ energy release. It can make techni-O-helium (as well as any other form of O-helium) an interesting candidate, which

might explain the difference between the positive result of DAMA/NaI (DAMA/Libra) and negative results of other experiments on direct dark matter search.

The destruction of techni-O-helium by cosmic rays in the Galaxy releases free charged technileptons, which can be accelerated and contribute to the flux of cosmic rays. In this context, the search for techniparticles at accelerators and in cosmic rays acquires the meaning of a crucial test for the existence of the basic components of the composite dark matter. At accelerators, techniparticles would look like stable doubly charged heavy leptons, while in cosmic rays, they represent a heavy -2 charge component with anomalously low ratio of electric charge to mass. If it has the same energy spectrum as ordinary cosmic rays, it can be observed in the PAMELA experiment.

To conclude, the minimal walking technicolor cosmology can give a robust cosmological scenario of composite dark matter, giving rise to a set of exciting observable effects.

\section{Acknowledgements}

We express our gratitude to P. Belli, K.M. Belotsky, J. Filippini, A.G. Mayorov, P. Picozza, V.A. Rubakov and E.Yu. Soldatov for important comments and for useful discussions. The work of CK was supported by the Marie Curie Fellowship under contract MEIF-CT- 
2006-039211.

[1] M. Y. Khlopov and C. Kouvaris, Phys. Rev. D 77, 065002 (2008) arXiv:0710.2189 [astro-ph]].

[2] F. Sannino and K. Tuominen, Phys. Rev. D 71, 051901 (2005); arXiv:hep-ph/0405209.

[3] D. K. Hong, S. D. H. Hsu and F. Sannino, Phys. Lett. B 597, 89 (2004); arXiv:hep-ph/0406200.

[4] D. D. Dietrich, F. Sannino and K. Tuominen, Phys. Rev. D 72, 055001 (2005); arXiv:hep-ph/0505059.

[5] D. D. Dietrich and F. Sannino, Phys. Rev. D 75, 085018 (2007) arXiv:hep-ph/0611341.

[6] S. B. Gudnason, T. A. Ryttov and F. Sannino, Phys. Rev. D 76, 015005 (2007) arXiv:hep-ph/0612230.

[7] S. B. Gudnason, C. Kouvaris and F. Sannino, Phys. Rev. D 73, 115003 (2006); arXiv:hep-ph/0603014.

[8] R. Foadi, M. T. Frandsen, T. A. Ryttov and F. Sannino, Phys. Rev. D 76, 055005 (2007) arXiv:0706.1696 [hep-ph]].

[9] D. D. Dietrich and C. Kouvaris, arXiv:0805.1503 [hep-ph].

[10] S. Catterall and F. Sannino, Phys. Rev. D 76, 034504 (2007) [arXiv:0705.1664 [hep-lat]].

[11] L. Del Debbio, M. T. Frandsen, H. Panagopoulos and F. Sannino, arXiv:0802.0891 [hep-lat].

[12] L. Del Debbio, A. Patella and C. Pica, arXiv:0805.2058 [hep-lat].

[13] S. B. Gudnason, C. Kouvaris and F. Sannino, Phys. Rev. D 74, 095008 (2006); arXiv:hep-ph/0608055.

[14] C. Kouvaris, Phys. Rev. D 76, 015011 (2007) arXiv:hep-ph/0703266.

[15] K. Kainulainen, K. Tuominen and J. Virkajarvi, Phys. Rev. D 75, 085003 (2007) arXiv:hep-ph/0612247.

[16] S. L. Glashow, arXiv:hep-ph/0504287.

[17] D. Fargion and M. Khlopov, arXiv:hep-ph/0507087.

[18] D. Fargion, M. Khlopov and C. A. Stephan, Class. Quant. Grav. 23, 7305 (2006) arXiv:astro-ph/0511789.

[19] M. Y. Khlopov and C. A. Stephan, arXiv:astro-ph/0603187.

[20] A. Connes, "Noncommutative geometry", Academic Press, London and San Diego, 1994.

[21] C. A. Stephan, J. Phys. A 39, 9657 (2006) arXiv:hep-th/0509213. 
[22] M. Y. Khlopov, Pisma Zh. Eksp. Teor. Fiz. 83, 3 (2006) [JETP Lett. 83, 1 (2006)] arXiv:astro-ph/0511796].

[23] K. Belotsky, M. Khlopov and K. Shibaev, arXiv:astro-ph/0602261.

[24] K. M. Belotsky, M. Y. Khlopov and K. I. Shibaev, Grav. Cosmol. 12, 93 (2006) arXiv:astro-ph/0604518.

[25] K. M. Belotsky, M. Y. Khlopov and K. I. Shibaev, Stable quarks of the 4th family? invited contribution to the book "The Physics of Quarks: New Research." to be published by NOVA, 2008 .

[26] K. M. Belotsky, M. Y. Khlopov, K. I. Shibaev, D. Fargion, R. V. Konoplich and M. G. Ryskin, Grav. Cosmol. 11 (2005) 3.

[27] M. Y. Khlopov, arXiv:astro-ph/0607048.

[28] M. Y. Khlopov, Prepared for 10th Workshop on What Comes Beyond the Standard Model, Bled, Slovenia, 17-27 Jul 2007

[29] M. Y. Khlopov, arXiv:0801.0167 [astro-ph].

[30] M. Y. Khlopov, arXiv:0801.0169 [astro-ph].

[31] P. Mueller, Phys.Rev.Lett. 92, 22501 2004; arXiv:nucl-ex/0302025.

[32] D. S. Akerib et al. [CDMS Collaboration], Phys. Rev. Lett. 96, 011302 (2006) arXiv:astro-ph/0509259.

[33] Z. Ahmed et al. [CDMS Collaboration], arXiv:0802.3530 [astro-ph].

[34] R. Bernabei et al., Riv. Nuovo Cim. 26N1, 1 (2003) [arXiv:astro-ph/0307403].

[35] R. Bernabei et al. [DAMA Collaboration], arXiv:0804.2741 [astro-ph].

[36] A. D. Dolgov and F. Takahashi, Nucl. Phys. B 688 (2004) 189 arXiv:hep-ph/0402066.

[37] J. D. Lewin and P. F. Smith, Astropart. Phys. 6, 87 (1996).

[38] M. Y. Khlopov, "Cosmoparticle physics," Singapore: World Scientific (1999) 577 p

[39] K. A. Olive and E. D. Skillman, Astrophys. J. 617, 29 (2004) arXiv:astro-ph/0405588.

[40] R. N. Cahn and S. L. Glashow, Science 213, 607 (1981).

[41] B. D. Wandelt et al., "Self-interacting dark matter," arXiv:astro-ph/0006344 P. C. McGuire and P. J. Steinhardt, "Cracking open the window for strongly interacting massive particles as the halo dark matter," arXiv:astro-ph/0105567; G. Zaharijas and G. R. Farrar, Phys. Rev. D72, 083502 (2005); arXiv:astro-ph/0406531.

[42] C. B. Dover, T. K. Gaisser and G. Steigman, Phys. Rev. Lett. 42, 1117 (1979); S. Wolfram, 
Phys. Lett. B82, 65 (1979); G. D. Starkman et al., Phys. Rev. D41, 3594 (1990); D. Javorsek et al., Phys. Rev. Lett. 87, 231804 (2001); S. Mitra, Phys. Rev. D70, 103517 (2004); arXiv:astro-ph/0408341. Mack, G.D.; Beacom, J.F.; Bertone, G. Phys Rev 2007, vol D76, 043523; arXiv:0705.4298 [astro-ph].

[43] D. McCammon et al., Nucl. Instr. Meth. A370, 266 (1996); D. McCammon et al., Astrophys. J. 576, 188 (2002); arXiv:astro-ph/0205012.

[44] K. Belotsky, Yu. Bunkov, H. Godfrin, M. Khlopov and R. Konoplich, "He-3 experimentum crucis for dark matter puzzles," arXiv:astro-ph/0606350. 